Operative Techniken

Oper Orthop Traumatol 2019 $\cdot 31: 284-292$ https://doi.org/10.1007/s00064-019-0608-6 Eingegangen: 18. Oktober 2018

Überarbeitet: 20. Januar 2019

Angenommen: 12. März 2019

Online publiziert: 3. Juni 2019

() Der/die Autor(en) 2019

\section{Redaktion}

K. Dresing, Göttingen

Zeichner

R. Himmelhan, Mannheim

\section{Vorbemerkungen}

Das Vorliegen einer Osteoporose manifestiert sich häufig im Rahmen einer Wirbelkörperfraktur [1]. Bei Vorliegen von instabilen sowie sekundär kyphosierten Frakturen ist die dorsale Instrumentation/Spondylodese oft die Therapie der Wahl [2-4]. Aufgrund schlechter Knochenqualität kann die Verankerung der hierfür benötigten Pedikelschrauben im Wirbelkörper jedoch schwierig sein und mit dieser Problematik einhergehend, zählen Schraubenlockerungen zu den häufigsten Versagensursachen dorsaler Instrumentationen [5-7].

In der Literatur werden verschiedene Ursachen für das Zustandekommen einer Schraubenlockerung beschrieben. Ein „Stress shielding“ könnte zu einem Remodeling des die Schraube umgebenden Knochens führen und somit eine Lockerung begünstigen [5]. Des Weiteren wurden Abrieb, Infektionen sowie lokale Überbelastung, beispielsweise durch fehlende Abstützung der ventralen Säule, als Risikofaktoren für eine Pedikelschraubenlockerung genannt $[5,8,9]$. Insbesondere bei älteren Patienten ist eine hohe Inzidenz von Schraubenlockerung beschrieben $[5,10]$. Diesbezüglich ist eine osteoporotische Knochenstruktur als größter Risikofaktur anzunehmen $[6,10,11]$, so dass die Inzidenz der Pedikelschraubenlockerung bei Patienten mit Osteoporose mit bis zu $60 \%$ angegeben wird [12].

Bei der operativen Versorgung osteoporotischer Wirbelkörperfrakturen müs-

Lukas Weiser · Stephan Sehmisch - Wolfgang Lehmann - Lennart Viezens

Klinik für Unfallchirurgie, Orthopädie und Plastische Chirurgie, Universitätsmedizin Göttingen, Göttingen, Deutschland

\title{
Techniken zur Steigerung der Pedikelschraubenstabilität im osteoporotischen Knochen
}

sen viele Faktoren berücksichtigt werden. An erster Stelle muss die verminderte Knochendichte detektiert werden, um eine adäquate Therapie gewährleisten zu können. Im Folgenden müssen die zur Verfügung stehenden Verfahren zur operativen Versorgung sowie der individuelle Patient und seine Begleitfaktoren, beispielsweise Vorerkrankungen, Körperbau/-gewicht und Statik der Wirbelsäule, evaluiert werden, um die individuelle Therapie des Patienten optimieren zu können.

\section{Operationsprinzip und -ziel}

Ziel des Eingriffs ist die Stabilisierung der Wirbelsäule, beispielsweise bei Vorliegen einer Wirbelkörperfraktur. Die eingebrachten Pedikelschrauben/Implantate müssen eine ausreichende Stabilität gewährleisten, um eine gegebenenfalls durchgeführte Reposition zu halten, eine Frakturheilung zu gewährleisten oder eine knöcherne Fusion zu ermöglichen.

\section{Vorteile}

- Bessere Vorbereitung auf den Eingriff

- Reduzierung der Komplikationsrate durch Verbesserung der Implantatstabilität

- Einfach zu erlernende und anzuwendende Techniken

- Frühfunktionelle Nachbehandlung mit orthograder Mobilisation des oftmals geriatrischen Patienten- kollektivs (auf das schwere Heben und Tragen von Lasten $>5 \mathrm{~kg}$ sollte verzichtet werden)

\section{Nachteile}

- Allgemeine Operationsrisiken

- Möglichkeit der Implantatfehllage und Verletzung benachbarter Strukturen

- Möglichkeit einer Zementembolie oder einer Zementleckage

\section{Indikationen}

- Osteoporotische Frakturen der Brustund Lendenwirbelsäule mit Instabilität, relevanter Achsabweichung, neurologischen Defiziten oder sekundärer Fehlstellung

- Stabilisation bei degenerativen Wirbelsäulenerkrankungen am osteoporotischen Knochen

- Stabilisation bei Tumorerkrankungen oder Infektionen im Bereich der Wirbelsäule und Vorliegen von osteoporotischer Knochenqualität

\section{Kontraindikationen}

- Allgemeine Kontraindikationen bezüglich Anästhesie und Operation

\section{Patientenaufklärung}

- Allgemeine Operationsrisiken

- Verletzungen benachbarter Strukturen, z. B. Spinalnerv, Dura, Myelon, Gefäße 
- Implantatfehllage, -bruch, -lockerung, -dislokation

- Zementleckage, Zementembolie, allergische Reaktionen

\section{Operationsvorbereitungen}

- Ausführliche Anamnese und Untersuchung des Patienten. Differenzierte Indikationsstellung anhand des radiologischen und klinischen Befunds sowie der Begleitfaktoren

- Bestimmung der Knochendichte in den zu instrumentierenden Wirbelkörpern mittels quantitativer Computertomographie (qCT) oder DXA-Messung („dual energy x-ray absorptiometry")

- Planung der Schraubenlänge und -dicke anhand der vorhandenen Bildgebung

\section{Instrumentarium}

- Pedikelschraubensystem

- Bildwandler

- Bei Zementaugmentation kanülierte, fenestrierte Schrauben und Augmentationskit mit Knochenzement

\section{Anästhesie und Lagerung}

- Vollnarkose

- Bauchlage auf einem röntgendurchlässigen Tisch

Oper Orthop Traumatol 2019 · 31:284-292 https://doi.org/10.1007/s00064-019-0608-6

(c) Der/die Autor(en) 2019

L. Weiser · S. Sehmisch · W. Lehmann · L. Viezens

\section{Techniken zur Steigerung der Pedikelschraubenstabilität im} osteoporotischen Knochen

\section{Zusammenfassung}

Operationsziel. Operative Stabilisation osteoporotischer Wirbelkörperfrakturen. Indikationen. Osteoporotische Frakturen der Brust- und Lendenwirbelsäule.

Kontraindikationen. Keine.

Operationstechnik. Präoperative Detektion der zu erwartenden Knochendichte.

Einbringen von Pedikelschrauben in speziellen Trajektorien, Augmentation des Schraubenlagers und Verwendung alternativer Implantate.

Weiterbehandlung. Frühfunktionelle, orthograde Mobilisation und ggf. Einleitung einer Osteoporosetherapie.

Ergebnisse. In einer biomechanischen Studie an humanen Kadaverwirbelkörpern konnte eine hochsignifikante Korrelation von Knochendichte und Pedikelschraubenstabilität nachgewiesen werden $\left(r^{2}=0,839 ; p<0,001\right)$. Des Weiteren konnte gezeigt werden, dass Pedikelschrauben bei einer Knochendichte $<80 \mathrm{mg} / \mathrm{cm}^{3}$ nur noch $60 \%$ der Versagenslast einer Pedikelschraube im Wirbelkörper mit normaler Knochendichte $\left(>120 \mathrm{mg} / \mathrm{cm}^{3}\right)$ aufweisen. Somit sind diese als nicht mehr ausreichend stabil anzusehen und es sollte über ein Verfahren zur zusätzlichen Stabilisation nachgedacht werden. In einem Dauerbelastungsversuch konnte gezeigt werden, dass eine Zementaugmentation im osteoporotischen Wirbelkörper zu einer Steigerung der Versagenslast um ca. 52\% führt (Versagenslast nicht augmentiert: $173 \mathrm{~N}$, augmentiert: $263 \mathrm{~N} ; p=0,001$ ). Weitere, von anderen Arbeitsgruppen durchgeführte Studien belegen den Effekt verschiedener Schraubentrajektorien und alternativer Implantate zur Verbesserung der Stabilität bei der Versorgung osteoporotischer Wirbelkörperfrakturen.

\section{Schlüsselwörter}

Osteoporose $\cdot$ Osteoporosefraktur $\cdot$ Wirbelsäule $\cdot$ Pedikelschraube $\cdot$ Knochendichte

\section{Techniques to increase pedicle screw stability in osteoporotic vertebrae}

\section{Abstract}

Objective. Operative stabilisation of osteoporotic vertebral fractures.

Indications. Fractures of the thoracic and lumbar spine.

Contraindications. None.

Surgical technique. Preoperative determination of expected bone mineral density. Implantation of pedicle screws in special trajectories, cement augmentation of the screws and use of alternative implants. Postoperative management. Early functional mobilisation and initiation of osteoporosis therapy if indicated.

Results. A biomechanical cadaver study with human vertebral bodies was performed. There was a highly significant correlation between bone mineral density and the fatigue load $\left(r^{2}=0.839, p<0.001\right)$. Specimens with bone mineral density below
$80 \mathrm{mg} / \mathrm{cm}^{3}$ only reached $60 \%$ of the fatigue load compared to the specimens with adequate bone quality $\left(>120 \mathrm{mg} / \mathrm{cm}^{3}\right)$ and therefore stability mightbe insufficient and an additional stabilisation should be considered. In osteoporotic vertebrae, the fatigue strength of cement augmented screws was increased by $52 \%$ compared to the non-augmented screws (fatigue load non-augmented: $173 \mathrm{~N}$, augmented: $263 \mathrm{~N}$; $p=0.001$ ). Studies conducted by other research groups have demonstrated the effect of various screw trajectories and alternative implants to improve stability in the treatment of osteoporotic vertebral body fractures.

\section{Keywords}

Osteoporosis - Osteoporotic fracture - Spine . Pedicle screw · Bone mineral density 


\section{Operative Techniken}

\section{Operationstechnik}

(• Abb. 1, 2, 3, 4, 5, 6, 7).

\section{Zementaugmentation}

a

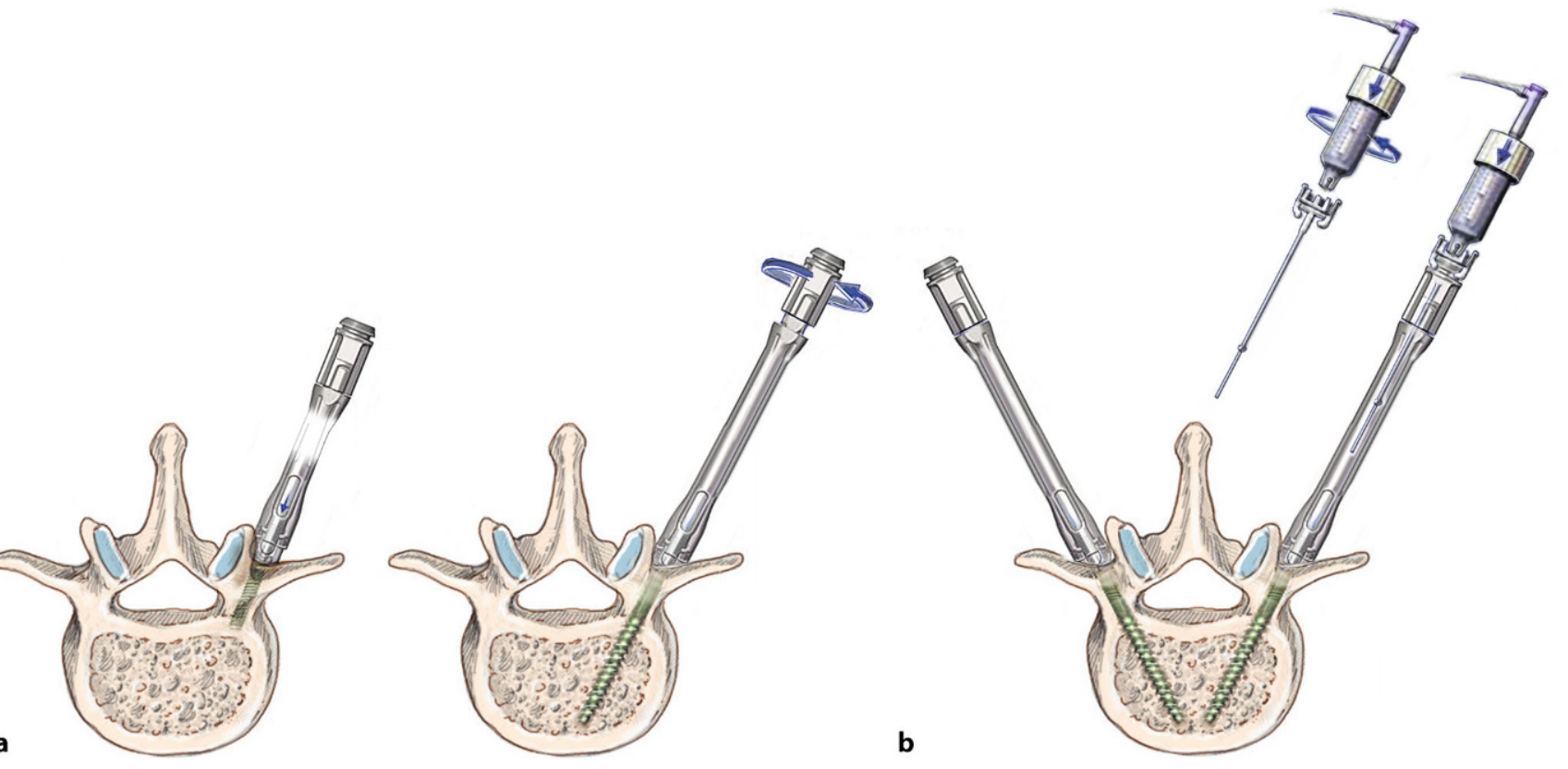

Abb. $1 \Delta$ a Bei der Zementaugmentation von Pedikelschrauben werden zunächst kanülierte, fenestrierte Pedikelschrauben in den Wirbelkörper eingebracht. Mittels Bildwandler ist zu kontrollieren, dass eine exakte Lage im Wirbelkörper vorliegt, um die Möglichkeit einer Leckage zu minimieren. b Anschließend kann über eine spezielle Augmentationskanüle Knochenzement durch die Schraube appliziert werden. Aus unserer Erfahrung und aus der existierenden Literatur ist die Verwendung von 1-1,5 ml Zement pro Schraube ausreichend, um eine adäquate Stabilitätssteigerung zu erreichen und gleichzeitig das Risiko für eine Zementleckage oder Embolie sowie für eine Anschlussfraktur zu minimieren [13] 


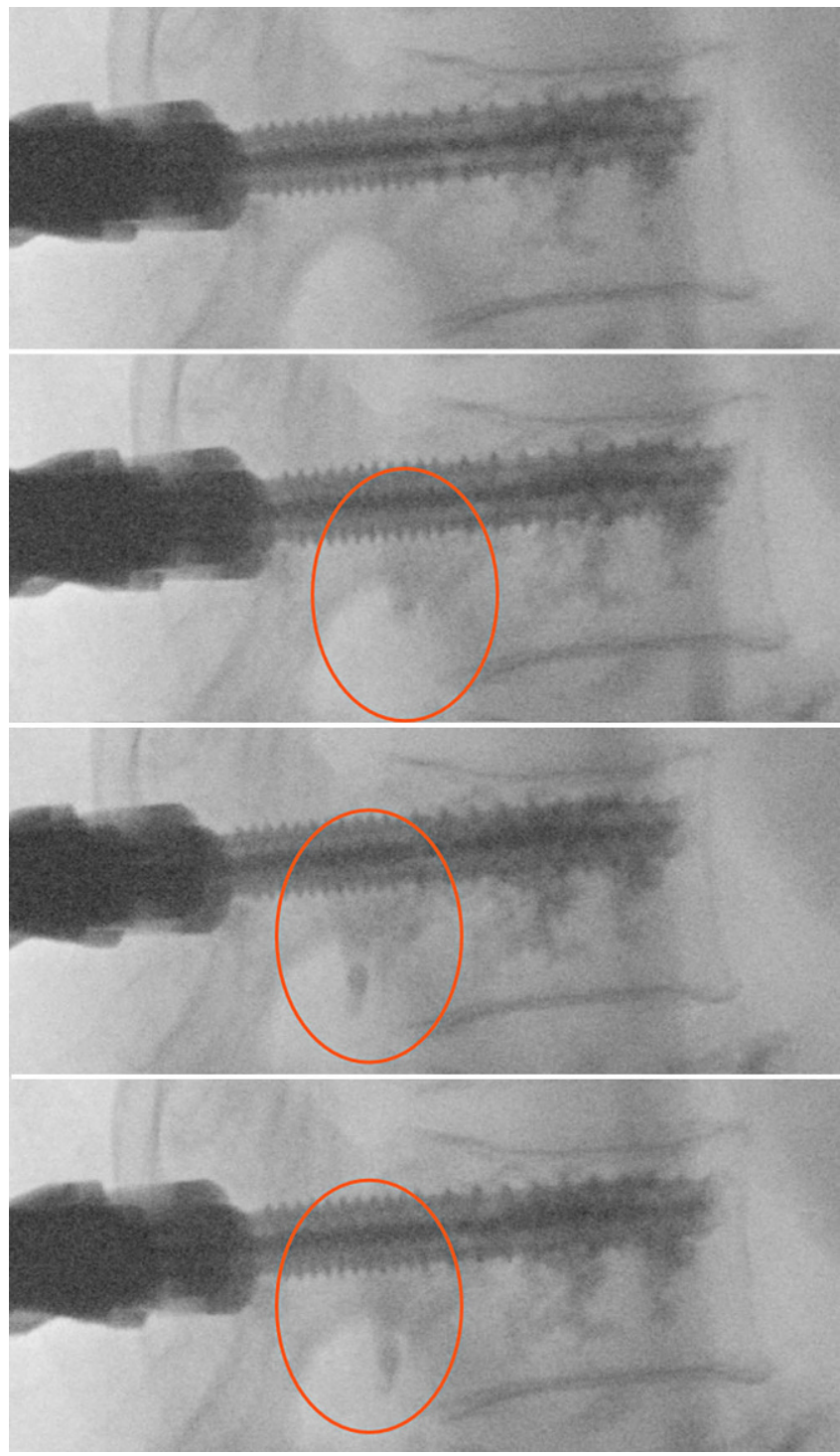

Abb. $2 \triangleleft$ Während der Augmentation sollten regelmäßige radiologische Kontrollen erfolgen, um eine regelrechte $Z \mathrm{e}$ mentverteilung im Wirbelkörper sicherzustellen. Bei ersten Anzeichen für eine Leckage (roter Kreis) sollte die Augmentation der betroffenen Schraube sofort abgebrochen werden 


\section{Alternative Schraubentrajektorien}
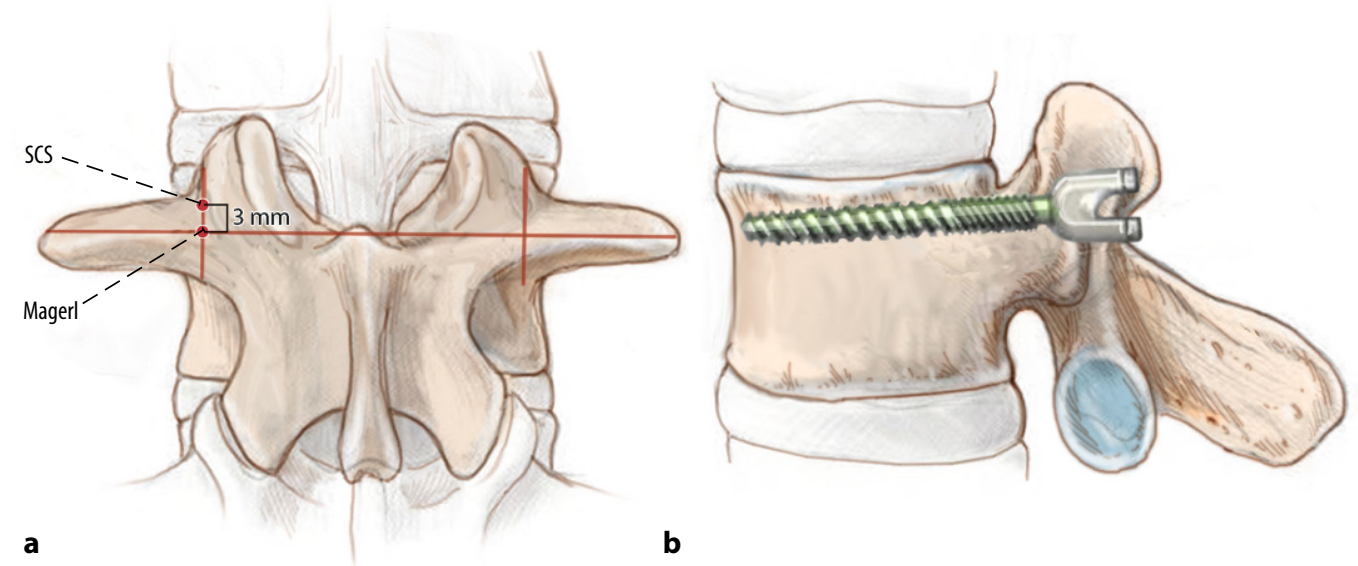

b

Abb. 3 ॥ In Anbetracht der hohen Rate an möglichen Komplikationen im Rahmen der Zementaugmentation, ist die Einbringung von Pedikelschrauben in alternativen Trajektorien eine gute Alternative zur Verbesserung der Schraubenstabilität im osteoporotischen Knochen. Bei der "Superior-cortical-screw"-(SCS-)Technik, wird die lumbale Pedikelschraube ca. $3 \mathrm{~mm}$ kranial des klassischen Eintrittspunkts nach Magerl eingebracht. Durch eine höhere Knochendichte im kranialen Anteil des Wirbelkörpers kann eine um bis zu 22,4\% gesteigerte Ausrisskraft verglichen mit Pedikelschrauben, die in Magerl-Technik eingebracht wurden, erreicht werden [14]. Es sollte darauf geachtet werden, dass die Schraubentrajektorie möglichst parallel zur Deckplatte verläuft und nicht von kaudal nach kranial ansteigt, da ansonsten eine Stressspitze an der Schraubenspitze erzeugt werden könnte

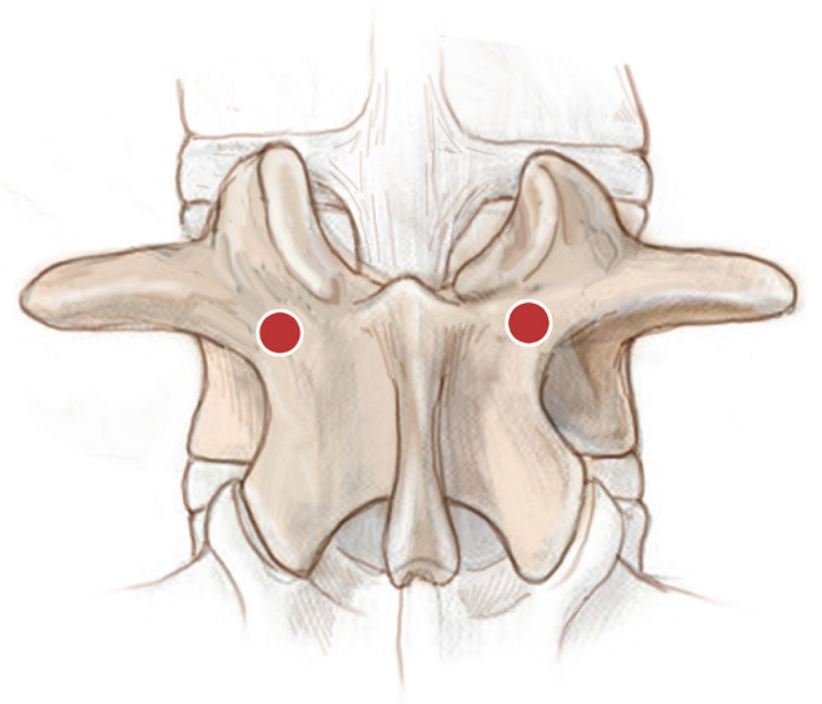

Abb. 4 A Die "Corticle-bone-trajectory“-(CBT-)Technik ist ein weiteres Verfahren, um die Schraubenstabilität im osteoporotischen Knochen zu verbessern. Da das Vorliegen einer Osteoporose sich wesentlich auf die spongiöse Knochenstruktur auswirkt, wird bei dieser Technik versucht, den kortikalen Schraubenhalt zu optimieren. Der Eintrittspunkt liegt im lateralen Bereich der Pars interarticularis, etwa bei der 5-Uhr-Position für den linken Pedikel und 7-Uhr-Position für den rechten Pedikel (rote Punkte) 

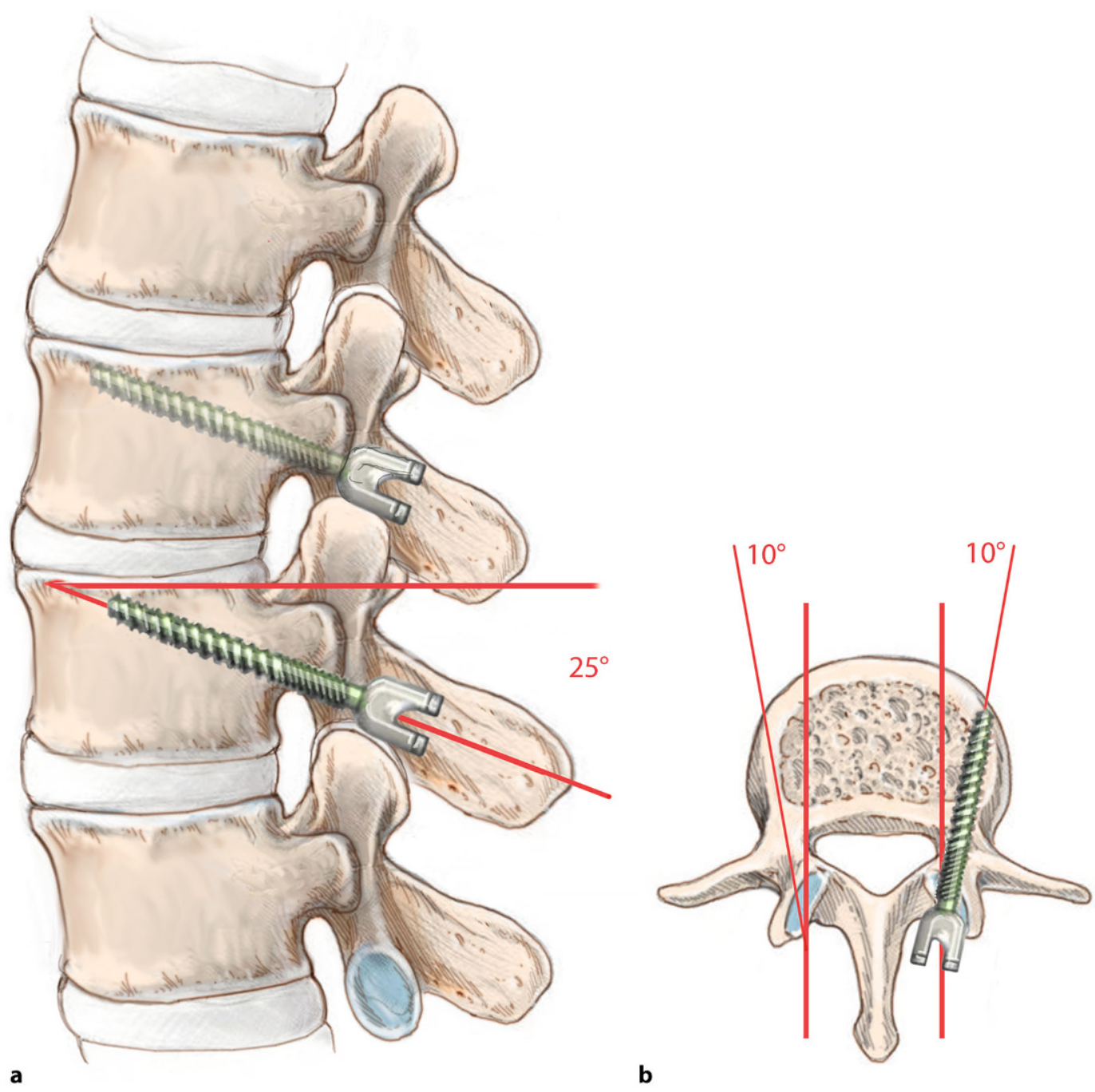

Abb. $5 \varangle$ Anschließend wird die Schraube in $25^{\circ}$-Ausrichtung nach kranial (a) und in ca. $10^{\circ}$-Ausrichtung nach lateral (b) eingebracht. Zur Erleichterung kann der Eintrittspunkt mit einer „High-speed"-Fräse eröffnet werden. Verglichen mit konventionellen Pedikelschrauben weisen die "Corticle-bone-trajectory"-Schrauben eine höhere Stabilität im Knochen und eine stabilere kortikale Verankerung auf $[15,16]$ 


\section{Operative Techniken}

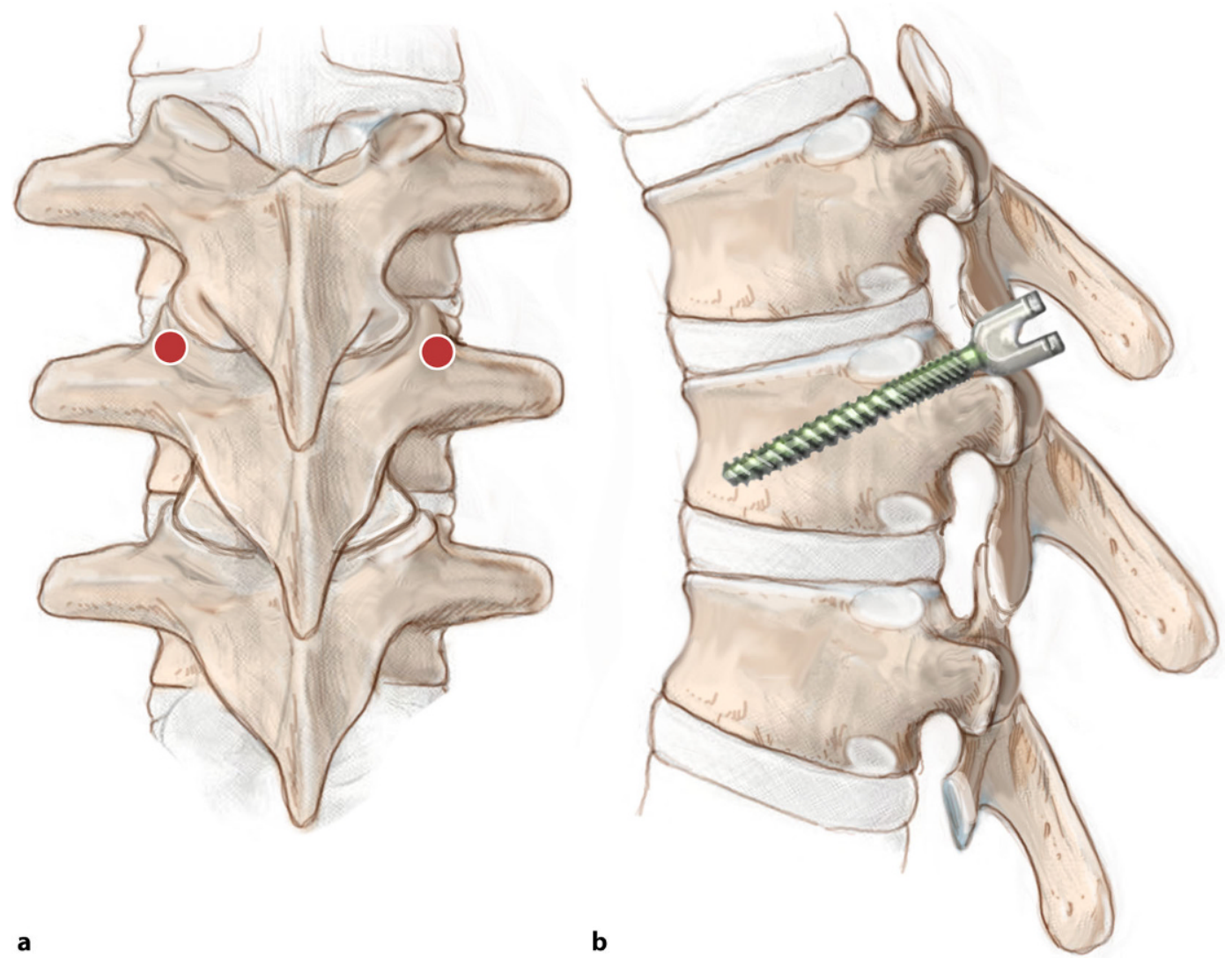

Abb. 6 ॥ Bei der Cauda-Technik werden die Pedikelschrauben, insbesondere im Bereich der Brustwirbelsäule, in der anatomischen Achse des Pedikels eingebracht (rote Punkte). Die führt im Bereich der Brustwirbelkörper zu einer ca. $20^{\circ}$ messenden cephalokaudalen Ausrichtung der Schrauben. Hierdurch entsteht eine längere Schraubentrajektorie verglichen mit deckplattenparallelen Schrauben. Des Weiteren kann der kortikale Kontakt posterosuperior und inferoanterior erhöht werden und somit die Schraubenstabilität optimiert werden. Auch bei gelockerten Schrauben, kann diese Technik als Revisionslösung in Erwägung gezogen werden. Der Eintrittspunkt für die Cauda-Schrauben befindet sich am kranialen Übergang von Lamina zu Proc. transversus am aufsteigenden Schenkel; die Schraubenachse verläuft in Abhängigkeit des Wirbelkörpers $0-10^{\circ}$ nach medial gerichtet und etwa $20^{\circ}$ nach kaudal gerichtet. Aufgrund der nach kaudal ausgerichteten Trajektorie sollten bei dieser Technik lediglich polyaxiale Pedikelschrauben verwendet werden 
Wahl der Implantate
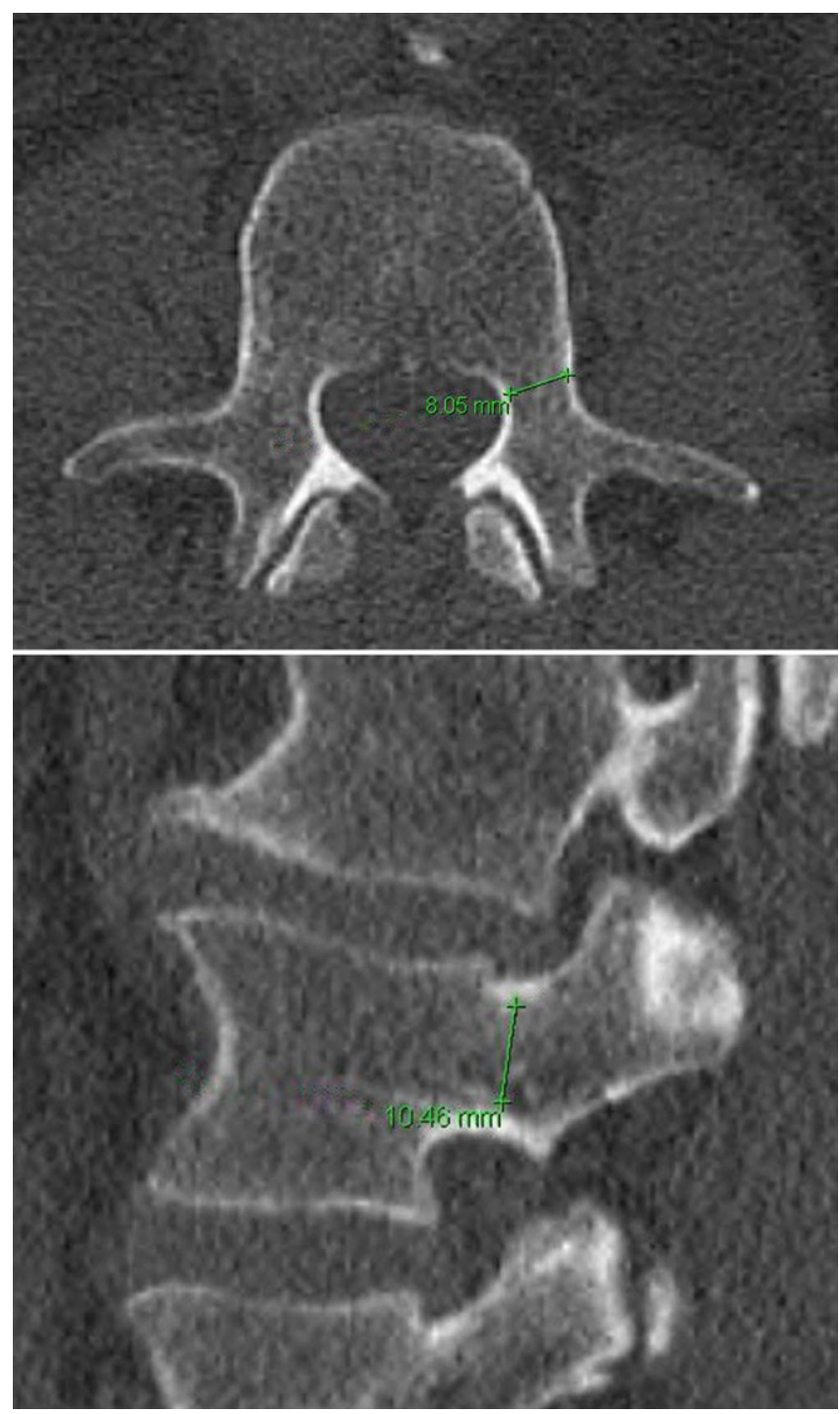

Abb. 7 ॥ Bei der Wahl der Implantate hat sich gezeigt, dass der Schraubendurchmesser die Pedikelschraubenstabilität signifikant beeinflussen kann. Es ist daher zu empfehlen, im Rahmen der präoperativen Bildgebung die Pedikelweiten der jeweilszu instrumentierenden Wirbelkörper auszumessen, um eine möglichst dicke Schraube einzubringen. Des Weiteren gibt es verschiedene Schraubendesigns, etwa mit zylindrischer Form oder mit einer Änderung der Gewindegänge, die eine stabilere Verankerung der Schraube im Wirbelkörper bzw. im Pedikel gewährleisten können. Eine weitere Alternative ist die Verwendung von Laminahaken. Sie stützen sich an der hauptsächlich kortikal stabilisierten Lamina ab und gewähren so auch bei osteoporotischer Knochenqualität eine stabile Verankerung [17]

\section{Postoperative Behandlung}

- Direkt postoperative Kontrolle der peripheren Durchblutung, Motorik und Sensibilität

- Mobilisation ab dem 1. postoperativen Tag

- Radiologische Kontrolle mittels konventionellen Röntgenbilds und ggf. Computertomographie (CT)

- Funktionelle Nachbehandlung mit orthograder Mobilisation unter Vermeidung des Hebens schwerer Lasten $>5 \mathrm{~kg}$

- Medikamentöse Behandlung der Osteoporose und Kontrollen im Verlauf

- Ggf. Materialentfernung im Verlauf (>6 Monate)

\section{Fehler, Gefahren, Komplikationen}

- Zementleckage und -embolie

- Verletzungen benachbarter Strukturen, insbesondere Spinalnerv, Dura, Myelon

- Materialfehllage, Materiallockerung

\section{Ergebnisse}

In einer von den Autoren durchgeführten biomechanischen Studie an humanen Kadaverwirbelkörpern wurde ein Dauerbelastungstest von konventionell eingebrachten Pedikelschrauben in Wirbelkörpern unterschiedlicher Knochendichte durchgeführt [18]. Es konnte eine hochsignifikante Korrelation von Knochendichte ( $\mathrm{qCT}$ ) und Pedikelschraubenstabilität nachgewiesen werden $\left(\mathrm{r}^{2}=0,839 ; p<0,001\right)$. Des Weiteren konnte gezeigt werden, dass Pedikelschrauben bei einer Knochendichte $<80 \mathrm{mg} / \mathrm{cm}^{3}$ nur noch $60 \%$ der Versagenslast und $45 \%$ der Zyklen bis zum Versagen einer Pedikelschraube im Wirbelkörper mit normaler Knochendichte („bone mineral density“: BMD $>120 \mathrm{mg} / \mathrm{cm}^{3}$ ) aufweisen und somit als nicht mehr ausreichend stabil anzusehen sind. Ist diesen Fällen ist dementsprechend über ein Verfahren zur Optimierung der Pedikelschraubenstabilität nachzudenken. In einer weiteren Studie erfolgte ein Dauerbe- 
lastungsversuch von augmentierten und nichtaugmentierten Pedikelschrauben. Es konnte gezeigt werden, dass eine Zementaugmentation im osteoporotischen Wirbelkörper zu einer Steigerung der Versagenslast um circa $52 \%$ führt (Versagenslast nichtaugmentiert: $173 \mathrm{~N}$, augmentiert: $263 \mathrm{~N} ; p=0,001)$. Im osteopenen Wirbelkörper zeigte sich eine Steigerung der Versagenslast um 33\%, während sich in den Wirbelkörpern mit normaler Knochendichte kein Unterschied in den Versagenslasten der augmentierten und nichtaugmentierten Pedikelschrauben zeigte [19].

\section{Korrespondenzadresse}

\section{Dr. Lukas Weiser}

Klinik für Unfallchirurgie, Orthopädie und Plastische Chirurgie, Universitätsmedizin Göttingen

Robert-Koch-Straße 40, 37099 Göttingen, Deutschland

Lukas.Weiser@med.uni-goettingen.de

\section{Einhaltung ethischer Richtlinien}

Interessenkonflikt. L. Weiser, S. Sehmisch, W. Lehmann und L. Viezens geben an, dass kein Interessenkonflikt besteht.

Für diesen Beitrag wurden von den Autoren keine Studien an Menschen oder Tieren durchgeführt. Für die aufgeführten Studien gelten die jeweils dort angegebenen ethischen Richtlinien.

Open Access Dieser Artikel wird unter der Creative Commons Namensnennung 4.0 International Lizenz (http://creativecommons.org/licenses/by/4.0/deed. de) veröffentlicht, welche die Nutzung, Vervielfältigung, Bearbeitung, Verbreitung und Wiedergabe in jeglichem Medium und Format erlaubt, sofern Sie den/die ursprünglichen Autor(en) und die Quelle ordnungsgemäßnennen, einen Link zur Creative Commons Lizenz beifügen und angeben, ob Änderungen vorgenommen wurden.

\section{Literatur}

1. Burge R, Dawson-Hughes B, Solomon DH, Wong JB, King A, Tosteson A (2007) Incidence and economic burden of osteoporosis-related fractures in the United States, 2005-2025. J Bone Miner Res 22(3):465-475

2. Cho W, Cho SK, Wu C (2010) The biomechanics of pedicle screw-based instrumentation. J Bone Joint Surg Br 92(8):1061-1065

3. Gaines RW Jr. (2000) The use of pedicle-screw internal fixation for the operative treatment of spinal disorders. J Bone Joint Surg Am 82A(10):1458-1476
4. Wood KB, Li W, Lebl DR, Ploumis A (2014) Management of thoracolumbar spine fractures. Spine J 14(1):145-164

5. Galbusera F, Volkheimer D, Reitmaier S, BergerRoscher N, Kienle A, Wilke HJ (2015) Pedicle screw loosening: a clinically relevant complication? Eur Spine J 24(5):1005-1016

6. Halvorson TL, Kelley LA, Thomas KA, Whitecloud TS 3rd, Cook SD (1994) Effects of bone mineral density on pedicle screw fixation. Spine 19(21):2415-2420

7. Soshi S, Shiba R, Kondo H, Murota K (1991) An experimental study on transpedicular screw fixation in relation to osteoporosis of the lumbar spine. Spine 16(11):1335-1341

8. Hallab NJ, Cunningham BW, Jacobs JJ (2003) Spinal implant debris-induced osteolysis. Spine 28(20):125-138

9. Villa T, La Barbera L, Galbusera F (2014) Comparative analysis of international standards for the fatigue testing of posterior spinal fixation systems. Spine J 14(4):695-704

10. Wu ZX, Gong FT, Liu L, Ma ZS, Zhang Y, Zhao X, Yang M, Lei W, Sang HX (2012) A comparative study on screw loosening in osteoporotic lumbar spine fusion between expandable and conventional pedicle screws. Arch Orthop Trauma Surg 132(4):471-476

11. Paxinos $O$, Tsitsopoulos PP, Zindrick MR, Voronov LI, Lorenz MA, Havey RM, Patwardhan AG (2010) Evaluation of pullout strength and failure mechanism of posterior instrumentation in normal and osteopenic thoracic vertebrae. J Neurosurg Spine 13(4):469-476

12. El Saman A, Meier S, Sander A, Kelm A, Marzi I, Laurer H (2013) Reduced loosening rate and loss of correction following posterior stabilization with or without PMMA augmentation of pedicle screws in vertebral fractures in the elderly. Eur J Trauma Emerg Surg 39(5):455-460

13. Pishnamaz $M$, Lange $H$, Herren $C, N a H S$, Lichte P, Hildebrand F, Pape HC, Kobbe P (2018) The quantity of bone cement influences the anchorage of augmented pedicle screws in the osteoporotic spine: a biomechanical human cadaveric study. Clin Biomech (Bristol, Avon) 52:14-19

14. Yu T, Zhang X, Liu J, Li S, Shan Z, Fan S, Zhao F (2018) Superior cortical screw in osteoporotic lumbar vertebrae: a biomechanics and microstructurebased study. Clin Biomech (Bristol, Avon) 53:14-21

15. Matsukawa K, Taguchi E, Yato Y, Imabayashi H, Hosogane N, Asazuma T, Nemoto K (2015) Evaluation of the fixation strength of Pedicle screws using cortical bone trajectory: what is the ideal trajectory for optimal fixation? Spine 40(15):E873-E878

16. Matsukawa K, Yato $Y$, Imabayashi H, Hosogane N, Asazuma T, Nemoto K (2015) Biomechanical evaluation of the fixation strength of lumbar pedicle screws using cortical bone trajectory: a finite element study. J Neurosurg Spine 23(4):471-478

17. Wilke HJ, Kaiser D, Volkheimer D, Hackenbroch C, Puschel K, Rauschmann M (2016) A pedicle screw system and a lamina hook system provide simila primary and long-term stability: a biomechanical in vitro study with quasi-static and dynamic loading conditions. Eur Spine J 25(9):2919-2928

18. Weiser L, Huber G, Sellenschloh K, Viezens L, Puschel K, Morlock MM, Lehmann W (2017) Insufficient stability of pedicle screws in osteoporotic vertebrae: biomechanical correlation of bone $\mathrm{mi}-$ neral density and pedicle screw fixation strength. Eur Spine J 26(11):2891-2897
19. Weiser L, Huber G, Sellenschloh K, Viezens L, Puschel K, Morlock MM, Lehmann W (2018) Time to augment?! Impact of cement augmentation on pedicle screw fixation strength depending on bone mineral density. Eur Spine J 27(8):1964-1971 